\title{
Assessment of Low Doses Radiation Effects Using Tumor Markers Among Palestinian Radiation Workers
}

\author{
Areej Dahdol ${ }^{1,2}$ and Mohammad Hjouj ${ }^{*}$ \\ ${ }^{1}$ Salfit Health Center, Salfit, Palestine; ${ }^{2}$ Medical Imaging Department, Faculty of Health Professions, Al- \\ Quds University, Palestine
}

Received: December 23, 2019 / Accepted: February 1, 2020

\begin{abstract}
Understanding the impact of radiation on occupational workers has been a huge concern; particularly when it comes to cancer, which is considered a stochastic effect from radiation. This paper aims at investigating the effects of low radiation doses on tumor markers tests among Radiation Workers (RWs). It also aims at demonstrating the effect of independent variables, such exposed dose, smoking status, and the type of work on tumor markers. The researchers reviewed the collected database of tumor marker tests in four Governmental Hospitals between the period (2013- 2019) in order to trace the patterns of tumor marker over the years. The exposed dose record was taken from the Energy Department. Additionally, a questionnaire was distributed to acquire correlated independent variables with tumor markers records and $\mathbf{7 8}$ RWs participated in this study. Results indicated that after several years of tracing tumor markers, they all tend to increase in a normal range. Significantly, the Carcinoembryonic antigen (CEA) has increased by approximately $\mathbf{5 7 \%}$ in $\mathrm{RWs}$. Moreover, the smoking workers have a statistically significant change in CEA. Finally, the study has not shown any relationship between the radiation doses and tumor markers. In conclusion, to the best of the researchers' knowledge, this is the first study to investigate the correlated tumor markers with low radiation doses among occupational worker. The researchers believe that these findings will contribute to 'gap-filling' in low dose effects, and demonstrate the importance of laboratory medical tests in prediction of low doses effect. However, further investigations are needed in order to achieve more accurate results.
\end{abstract}

Keywords: Low dose, Radiation workers, Tumor markers, Cancer, Stochastic effects, Retrospective.

"Corresponding author: mhjouj@hotmail.com

\section{Introduction}

Occupational radiation workers (RWs) are more likely to receive low doses of radiation over a long period. The basic of low doses radiation effect is non-lethal mutations, with the biggest concern being the induction of cancer. This concern comes from the fact that some radiation risks are associated with dose by a linear, no-threshold model. In brief, linear means a proportional relationship between the dose and the risk. Additionally, any dose -no matter how small this does is-, which may cause some risks is called no-threshold. (Calabrese and O'Connor, 2014). Furthermore, the study reported that cancer in RWs has significantly increased over the years (Choi et al., 2013).

Several recent studies have shown evidence of cancer among occupational worker. One study used human's peripheral blood lymphocytes to assess the DNA damage. Antioxidant status shows that the chronic low dose exposure is an unavoidable hazard, which would lead to an oxidative stress, increased genomic instability, and a rise in cancer among RWs (Siama et al., 2019). Moreover, the incidence of thyroid cancer rates is higher among Korean RWs than the general population (Lee et al., 2018; Lee et al., 2019). Another study on health risks shows an increase in all of skin lesions, orthopedic illness, cataract, hypertension, and hypercholesterolemia in cardiac catheterization workers (Andreassiet al., 2016). One study, which focused on reliable cancer mortality risks from a low-dose in a nuclear industry, showed an evidence of stochastic effects and statistical significance for lung cancers (Qu et al., 2018). Based on that, it is crucial to provide the population with more intensive and protective measures, safe development tools, and acceptable tests for the early detection of cancer (Schiffman et al., 2015).

Tumor biomarker is one of the most important tools, referred as a secreted molecule in the blood arising out of a tumor in case of cancer presence. Although the field of radiation detection shows that, an assessment of absorbed dose using a protein with gene expression, will be considered as a good biomarker

This is an Open Access article distributed under the terms of the Creative Commons Attribution License (http://creativecommons.org/licenses/by/3.0/), which permits unrestricted use, distribution, and reproduction in any medium, provided the original work is properly cited. 
of radiation, the insufficient sensitivity and specificity of tumor marker tests generate positive results, yet this does not necessarily indicate the presence of cancer. This means that additional procedures, such as biopsy, x-rays, or etc., are needed in conjunction with these tests. (Sahoo, 2018; Kayaba, 2003; Rana et al., 2010).

Some tumor markers, such as CEA glycoprotein, are used for screening the elevated levels found in colorectal, breast, lung, or pancreatic cancer and, in smokers (Bhatt et al., 2010). However, it has special sensitivity among markers for colorectal cancer (Gao et al. 2018). Another marker is Cancer Antigen 19-9, commonly used for pancreatic cancer (Scarà, 2015). While the regular CA19.9 measurements show an improvement in early detection of biliary tract cancer, the last one will included in this study is a Carbohydrate Antigen 125, a tumor marker for ovarian cancer. However, study shows that a combination of CA125 and human epididymis protein 4 (HE4) improves the sensitivity and specificity of ovarian cancer (Wannhoff, 2019).

On the other hand, several studies have reported that multiple markers are more useful in the detection of some cancers. Some other studies reported that the combination of CA 19-9, CEA, and other markers will increase sensitivity and specificity in Gastric cancer. Moreover, other studies have shown that the combination contributes to diagnoses and prediction of pancreatic cancer. The combination of CA19-9 and CA125 has shown to encourage sensitivity for pancreatic cancer (Kotzevet al., 2018; Li et al., 2013; Meng et al., 2017; O'Brien et al., 2015).

Similarly, the impact of occupational radiation on some tumor markers, and the effect of high background radiation on them have been studied. A Study in 2019 in the field of Biomarkers indicates that, levels of serum $8-\mathrm{OHdG}$ which is a biomarker resulted in oxidative stress, were found to be significantly higher in interventional RWs (Gao et al., 2019). Likewise, a study in Ramsar (Iran) has shown that there is significant alteration in Cyfra 21, CEA, and Tag 72 levels due chronic exposure to high background radiation (Taeb et al., 2014).

The researchers believe that studying and investigating tumor markers might help highlight low radiation effects, especially cancer, among the workers are exposed to chronic low doses.

\section{Materials and Methods}

\section{Study Participants}

For the study approval, Al-Quds University sent letters including the purpose of the study to the Ministry of Health (MoH). Official permission was obtained to visit the Hospitals and distribute the questionnaires in addition to facilitate data collection procedures. A one-page participants' Consent Form was attached to the questionnaire. The researchers gave total freedom to accept or reject participation in this research. (78) RWs participated. Workers with diseases, such as (hepatic disorders, diabetes or any other kind of disease), were excluded for the accuracy of the results. A worker with diagnostic modalities that did not utilize ionizing radiation (like MRI, U/S) was also excluded.

\section{Data Collection}

After Institutional Review Board Approval, the researchers retrospectively reviewed a collected database of tumor marker tests in four Governmental Hospitals between 2013 and 2019 to trace the patterns of TM tests over the years. Commercial Architect kits had been used to measure the serum levels of CA125, CEA, and CA19.9 tumor markers. The test records are distributed as follows, 42 records of CEA, 45 records of CA 19.9, and 35 records of CA125 conducted over the years of the study. Independent variables, such as smoking status and the type of work, were reached and obtained by the distributed questionnaire, while the dose recorded was taken from a database of the Energy Department.

\section{Statistical Data Analysis}

Collected data were analyzed by using SPSS, Version 22 . Student's t-test was used for testing the equality of the means in the groups. P-value $<0.05$ was considered significant. Descriptive statistical analysis was used to determine the tumor marker behavior along several years.

\section{Results and Discussion}

\section{Assessment of Tumor Markers Test through Years}

At the beginning, the researchers tried to study whether the exposed dose had an effect on TMs by dividing RWs into two groups according to the exposed dose. One group had a registered radiation dose of about $1 \mathrm{mSv}$ or less, while the second had over $1 \mathrm{mSv}$. Then, the researchers compared their average means to each tumor markers. As noted, the average doses for each year from 2019, 2018, 2017, 2016, and 2015 with an annual average dose found to be at $0.50,0.67,0.77,0.5$, and 0.72 $\mathrm{mSv}$, respectively. The maximum doses were found to be 2.021 , $4.519,4.266,2.679,4.875 \mathrm{mSv}$, respectively. This means that the average dose for most RWs did not exceed the annual dose limit of $20 \mathrm{mSv}$ for occupational workers.

As shown in Table 1, the independent-sample t-test is used to investigate whether the exposed dose had an effect on TMs. The result shows no significant mean difference according to the exposed dose. Actually, the irregular readings of the exposed doses make the results inaccurate. Therefore, the researchers decided to explore what is going to happen to the TMs through years of exposure to the radiation.

Accordingly, one of the most important questions in this study is: what happens to TMs test over several years of work in the Radiation Department? According to Table 2 which represents the percentages of how TM tests of RWs behave over the years (from 2013 to 2019), the TM test pattern is expressed by scale levels (decrease, increase, and stable). However, the unstable state is ignored due to an unclear behavior. In addition the average number of each behavior according to specific year is demonstrated in (Figures 1, 2, and 3), for each type of tumor markers separately. These figures show how the average value was for each tumor marker and its behavior from the first year 
Table 1. Statistically significant relationship between tumor markers according to an exposed dose (independent T-test).

\begin{tabular}{|c|c|c|c|c|c|}
\hline Tumor Markers & Annual Exposure Dose & Mean & Std. Deviation & $\mathbf{T}$ & sig \\
\hline \multirow[t]{2}{*}{ CEA } & less than $1 \mathrm{msv}$ & 1.38 & 0.82 & -0.59 & 0.56 \\
\hline & more than $1 \mathrm{msv}$ & 7.42 & 6.40 & & \\
\hline \multirow[t]{2}{*}{ CA199 } & less than $1 \mathrm{msv}$ & 11.34 & 5.11 & -0.40 & 0.69 \\
\hline & more than $1 \mathrm{msv}$ & 1.38 & 0.82 & & \\
\hline \multirow[t]{2}{*}{ CA125 } & less than $1 \mathrm{msv}$ & 7.42 & 6.40 & 1.03 & 0.31 \\
\hline & more than $1 \mathrm{msv}$ & 11.34 & 5.11 & & \\
\hline
\end{tabular}

Table 2. Percentages of the behavior patterns of tumor markers during the years.

\begin{tabular}{lcccc}
\hline Tumor markers & & $*$ Decrease & $*$ Increase & $*$ Stable \\
\hline CEA & Percent $\%$ & $9 \%$ & $57 \%$ & $34 \%$ \\
Normal range $(\mathbf{0 - 5} \mathbf{~ n g / m l )}$ & & & & \\
\hline CA199 & Percent $\%$ & $31 \%$ & $46 \%$ & $23 \%$ \\
Normal range $(\mathbf{0 - 3 7} \mathbf{n g} / \mathbf{m l})$ & & & & \\
\hline CA125 & Percent $\%$ & $37 \%$ & $54 \%$ & \\
Normal range $(\mathbf{0 - 3 5} \mathbf{~ n g / m l )}$ & & & & \\
\hline
\end{tabular}

**Note that the increase, decrease and stable value was within normal range to all type of TM.

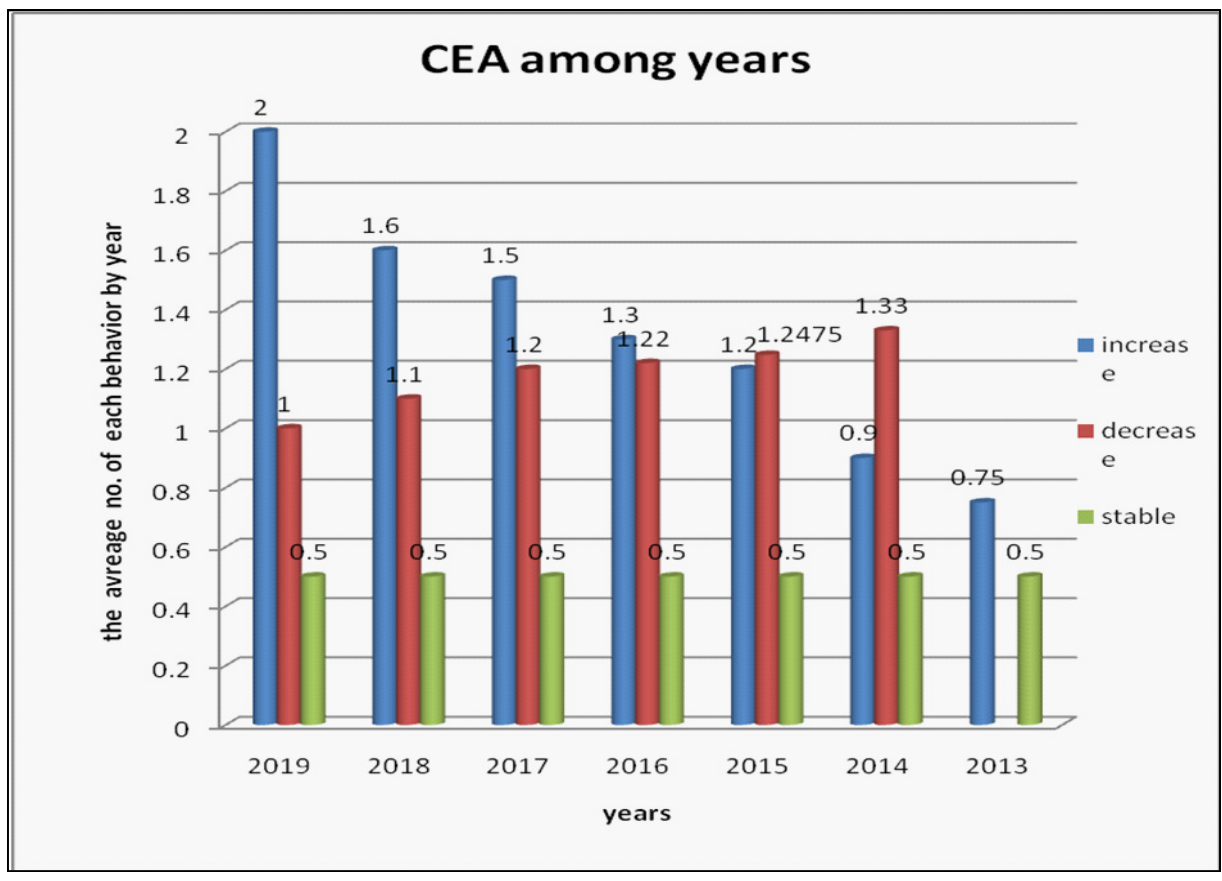

Figure 1. Demonstration of the average value for CEA behaviors by the year. The average CEA value was $(0.75 \mathrm{ng} / \mathrm{ml})$ in the first year (2013), which illustrates the average of the RWs who have an increase (which represent about $57 \%$ of the RWs, see table 2) and the average of increase became $(2.0 \mathrm{ng} / \mathrm{ml})$ in the last year (2019). This shows the rate of increase over the years. It is worth mention that this example applies to all behaviors. 


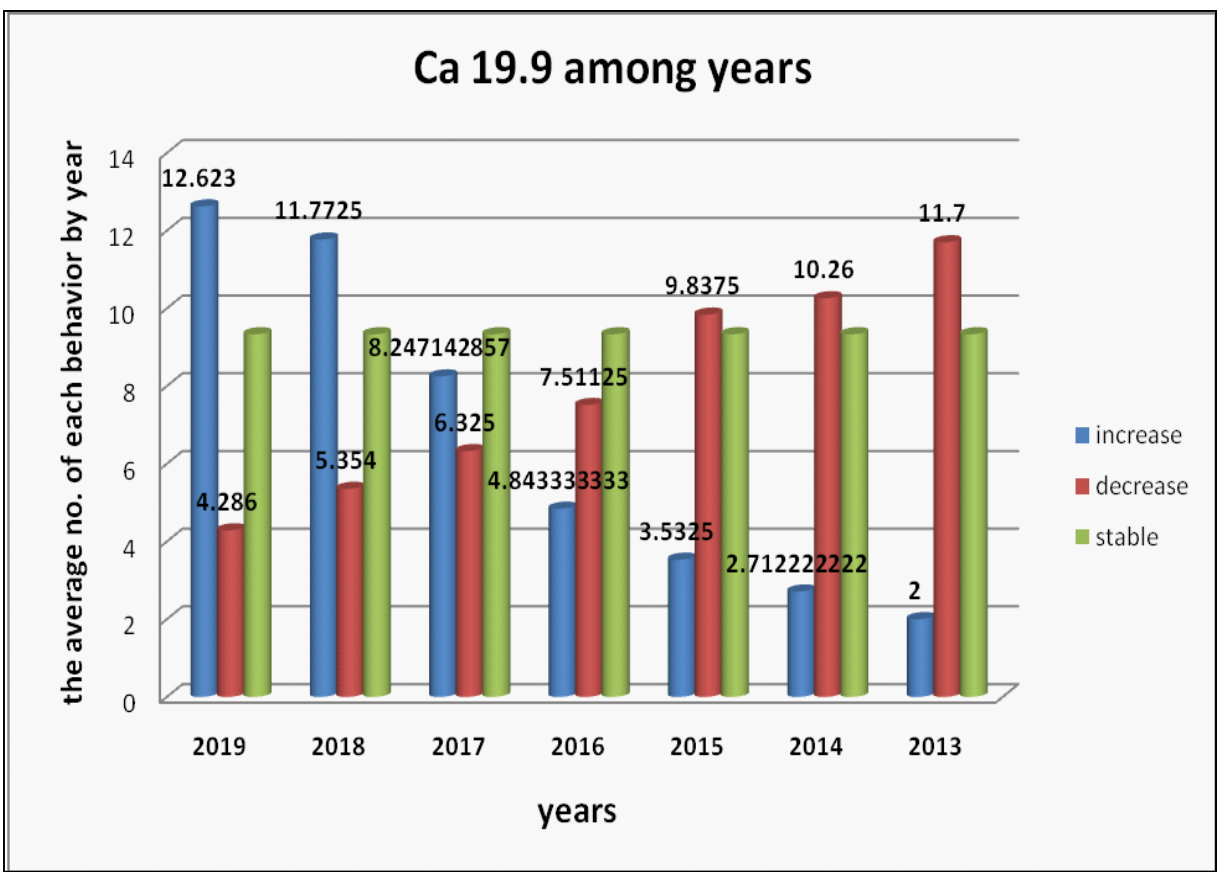

Figure 2. Demonstration of the average value for CA 19.9 behaviors by the year. The average value was $(2 \mathrm{ng} / \mathrm{ml})$ in the first year (2013), which illustrates the average of the RWs who have an increase (which represent about $46 \%$ of the RWs, see table 2), then the average of increase became $(12.0 \mathrm{ng} / \mathrm{ml})$ in the last year (2019). This shows the rate of increase over the years. It is worth mention that this example applies to all behaviors.

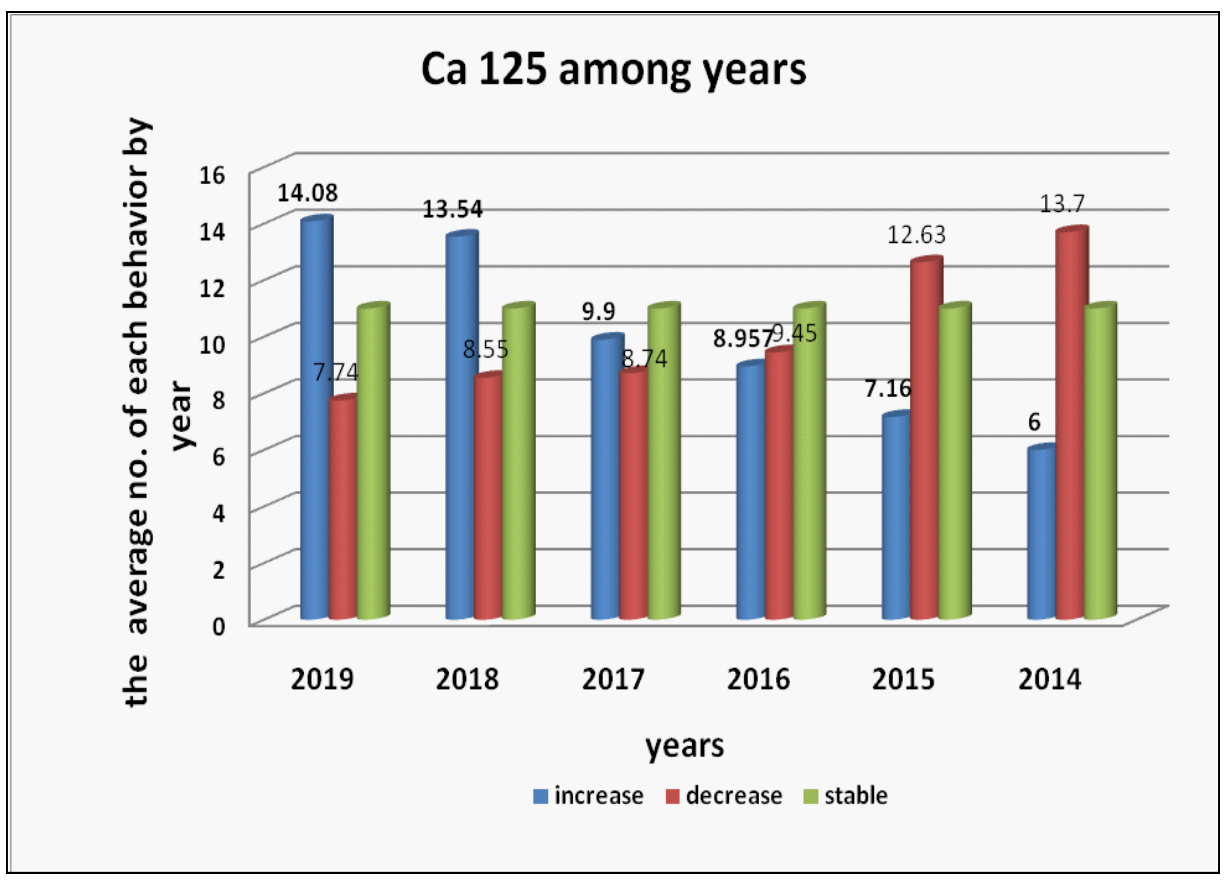

Figure 3. Demonstration of the average value for CA 125 behaviors by year. The average value was $(6.0 \mathrm{ng} / \mathrm{ml})$ in the first year $(2014)$, which illustrates the average of the RWs who have an increase (which represent about $54 \%$ of the RWs, see table 2), then the average of increase became $(14.0 \mathrm{ng} / \mathrm{ml})$ in the last year (2019). This shows the rate of increase over the years. It is worth mention that this example applies to all behaviors. 
Table 3. Statistically significant relationship between tumor markers according to smoking (Independent T-test).

\begin{tabular}{llcccc}
\hline Tumor Markers & smoking Status & Mean & Std. Deviation & T & sig \\
\hline CEA & Smoking & 1.71 & 0.84 & 2.88 & 0.01 \\
& Non-s moking & 1.21 & 0.59 & & \\
\hline CA199 & Smoking & 5.99 & 6.07 & 0.39 & 0.70 \\
& Non-s moking & 5.47 & 4.73 & & \\
\hline CA125 & Smoking & 11.14 & 4.54 & 0.77 & 0.45 \\
& Non-s moking & 10.12 & 5.37 & & \\
\hline
\end{tabular}

Table 4. Statistically significant relationship between tumor markers according to type of work (Independent T-test).

\begin{tabular}{llcccc}
\hline Tumor Markers & Type of Work & Mean & Std. Deviation & T & Sig \\
\hline CEA & CT , x-ray & 1.35 & 0.72 & -1.05 & 0.30 \\
& Interventional & 1.55 & 0.80 & & \\
\hline CA199 & CT , x-ray & 6.51 & 6.48 & 1.39 & 0.17 \\
& Interventional & 4.68 & 3.22 & & \\
\hline CA125 & CT , x-ray & 10.29 & 4.81 & -0.50 & 0.62 \\
& Interventional & 10.96 & 5.29 & & \\
\hline
\end{tabular}

to the last year.

As shown in Table 2, we found a 57\% increase in CEA TMs but within the normal range of 0-5 $\mathrm{ng} / \mathrm{ml}$ (Table 2, Figure 1). Only one RW had abnormal results in the CEA. This reminds us of the alteration occurrence in CEA levels in a high background radiation (Taeb et al., 2014). These findings may highlight CEA as the most sensitive TM to radiation, due to the highest percentage of increase among TMs, being more stable and having the lowest percentage of decrease over the years. The elevated CEA may appear in case of lung cancer as mentioned by Bhatt et al. (Bhatt et al., 2010). In fact, lungs are considered as one of the sensitive organs to radiation with a risk factor at $2.0 * 10$ -3 Sv-1 (Till and Grogan, 2008). Moreover, it is important to mention the effect of smoking in CEA (as will be discussed below), which may help in the interpretation of this increase. In any case, more investigation is needed to accurate the findings. However, CA-19.9 and CA125 have an increase of (46\%) and (54\%) within the normal range (figures 2 and 3 ) respectively. Additionally, there is a tendency for a decrease of $(31 \%)$ and $(37 \%)$, respectively, with a very low percentage of stability in CA-19.9 and CA125 as TMs. Overall, the increased pattern was the most behavior TMs have followed. This is despite the fact that, as mentioned in more than one study, the combination of TMs has shown to be an encouraging sensitivity, for detecting several types of cancer, such as colorectal cancer (Gao et al., 2018 ), Gastric cancer (Kotzev et al., 2018; Li et al., 2013), and pancreatic cancer. (Meng et al., 2017; O’Brien et al., 2015).

Lastly, the effect of low radiation on TMs behavior may have shown a little increase over the years, but a lot of investigation is needed to obtain results that are more accurate. It should be taken into account that this study is retrospective; therefore, the researchers have neither considered the systematic errors of equipment nor used any different types of equipment over the years, which, in fact, can affect the accuracy of the results.
The Effect of Tumor Markers by Independent Variables

The determination of how different variables can affect TMs is vital. It can help to understand how TMs are affected by radiation, after isolating the effects of other variables. The following tables illustrate significant differences at the level of $(\mathrm{p} \leq 0.05)$ due to TMs effects according to the independent variables, such as smoking status, and type of work. The researchers tested the hypotheses for each variable separately as follows:

\section{Smoking \& TMs}

The researchers compared the average means to each tumor marker for the two groups of RWs, smokers and non-smokers. The independent-sample t-test is used (table 3), the results show that only the means of CEA concentration between other markers have significant differences $(p=0.01)$. The significant differences were in favor of the RWs who are smokers, thus, coinciding with other studies that proved that smoking affect CEA markers (Bhatt et al., 2010; Nan et al., 2017). According to that, smoking may cause the effects of radiation on RWs to be worse. On the other hand; smoking does not affect the CA19-9 \& CA125. This was suspected because smoking is not considered as an important factor in these markers, according to previous studies.

\section{Work type \& TMS}

The researchers tried to find if there was a relationship between the two groups of workers who work on different devices, the X-ray / CT group and the interventional group. They conduct this by comparing the mean averages to each tumor marker for each group. Based on the results of the independentsample t-test (table 4), the means of the two groups were not significantly different. This may be attributed to the rotation system in the Radiation Departments (there is no specific RW 
to any type of equipment). It benefits to distribute a dose while working with high dose procedures (like interventional) among RWs. World Health Organization (WHO) reported, "Where higher doses are possible, careful use of worker rotations will reduce the risks" (World Health Organization, 2003).

\section{Conclusion}

The researchers believe that these findings will contribute to 'gap-filling' in low dose effects, and demonstrate the importance of laboratory medical tests in the prediction of low doses effect. The effect of radiation may be presented as little increase from years to years, as the increase is the most pattern that TMs is behaved. However, further investigation is still needed. It is important to repeat this study after few years in order to understand how radiation affects our bodies. Smoking workers have a significant difference in CEA tumor marker. A recommendation must be issued to reduce smoking when working with radiation because it might make the effects of the radiation worse for RWs. The rotation system does not only aim to train RWs to work with different types of procedures, but also helps distribute high doses to all workers, thus, making everyone in a safer environment. Surely this does not prevent tests from being inaccurate for the early detection of CA as mentioned earlier in previous studies. According to that, workers in this field may need more accurate tests, or we may consider radiation to be safer than what we really think. In light of this, studying tumor markers helps policymakers decide if they should approve policies to decrease the irradiation of occupational radiation work, or recommended better laboratory tests that monitor the chronic low doses effect on RWs.

Finally, the researchers state that due to chronic low doses of radiation received by occupational RWs, an effective remedial action program to protect this population should be of the highest concern.

\section{Conflict of Interest}

The authors declare no conflict of interest.

\section{Funding}

This work was not funded by any grant from public, commercial, or not-for-profit agencies.

\section{Acknowledgments}

Tha authors thank Mr. Marwan Zuhd for his help in statistical data analysis.

\section{References}

Andreassi MG, E Piccaluga, G Guagliumi, M Del Greco, F Gaita, and E Picano (2016) Occupational health risks in cardiac catheterization laboratory workers. Circ Cardio vasc Interv 9(4) : e003273.

Bhatt AN, R Mathur, A Farooque, A Verma, and BS Dwarakanath (2010) Cancer biomarkers-current perspectives. Indian J Med Res
132 (2): 129-149.

Calabrese EJ, and MK O'Connor (2014) Estimating risk of low radiation doses-a critical review of the BEIR VII Report and its use of the linear no-threshold (LNT) hypothesis. Radiat Res 182 (5): 463-474.

Choi KH, M Ha, W Lee, SS Hwang, M Jeong, YW Jin, H Kim, KY Lee, JE Lee, JW Kang, and H Kim (2013) Cancer risk in diagnostic radiation workers in Korea from 1996-2002. Int J Environ Res Public Health 10(1): 314-327.

Gao Y, P Wang, Z Wang, L Han, J Li, C Tian, F Zhao, J Wang, F Zhao, Q Zhang, and Y Lyu (2019) Serum 8-Hydroxy-2'-Deoxyguanosine Level as a Potential Biomarker of Oxidative DNA Damage Induced by Ionizing Radiation in Human Peripheral Blood. Dose Response 17(1): 1559325818820649.

Gao Y, J Wang, Y Zhou, S Sheng, SY Qian, and X Huo (2018) Evaluation of serum CEA, CA19-9, CA72-4, CA125 and ferritin as diagnostic markers and factors of clinical parameters for colorectal cancer. Sci Rep 8(1): 2732.

Kayaba H (2003) Tumor markers: essential diagnostic tools for radiologists. Nihon Igaku Hoshasen Gakkai zasshi. Nippon acta radiologica 63(4): 133-139.

Kotzev AI, and PV Draganov (2018) Carbohydrate Antigen 19-9, Carcinoembryonic Antigen, and Carbohydrate Antigen 72-4 in Gastric Cancer: Is the Old Band Still Playing?. Gastrointest Tumors 5(12): 1-13.

Lee WJ, Y Choi, S Ko, ES Cha, J Kim, YM Kim, KA Kong, S Seo, YJ Bang, and YW Ha (2018) Projected lifetime cancer risks from occupational radiation exposure among diagnostic medical radiation workers in South Korea. BMC cancer 18(1): 1206.

Lee WJ, DL Preston, ES Cha, S Ko, and H Lim (2019) Thyroid cancer risks among medical radiation workers in South Korea, 1996-2015. Environ Health 18(1): 19.

Li F, S Li, L Wei, X Liang, H Zhang, and J Liu (2013) The correlation between pre-operative serum tumor markers and lymph node metastasis in gastric cancer patients undergoing curative treatment. Biomarkers 18(7): 632-637.

Meng Q, S Shi, C Liang, D Liang, W Xu, and S Ji (2017) Diagnostic and prognostic value of carcinoembryonic antigen in pancreatic cancer: a systematic review and meta-analysis. Onco Targets Ther 10: 4591-4598. doi:10.2147/OTT.S145708.

Nan J, J Li, X Li, G Guo, X Wen, and Y Tian (2017) Preoperative serum carcinoembryonic antigen as a marker for predicting the outcome of three cancers. Biomarkers in cancer 9: 1179299X17690142.

O'Brien DP, NS Sandanayake, C Jenkinson, A Gentry-Maharaj, S Apostolidou, EO Fourkala, S Camuzeaux, O Blyuss, R Gunu, A Dawnay, and A Zaikin (2015) Serum CA19-9 is significantly upregulated up to 2 years before diagnosis with pancreatic cancer: implications for early disease detection. Clin Cancer Res 21(3): 622-631.

Qu SG, J Gao, B Tang, B Yu, YP Shen, and Y Tu (2018) Low-dose ionizing radiation increases the mortality risk of solid cancers in nuclear industry workers: A meta-analysis. MolClinOncol 8: 703711. https://doi.org/10.3892/mco.2018.1590

Rana S, R Kumar, S Sultana, and RK Sharma (2010) Radiation-induced biomarkers for the detection and assessment of absorbed radiation doses. J Pharm Bioallied Sci 2(3): 189-196. http://doi. org/10.4103/0975-7406.68500.

Sahoo TK (2018) Screening for early detection of cancer-Hope for cure. Oncol J India 2(2): 23.

Scarà S, P Bottoni, and R Scatena (2015) CA 19-9: biochemical and clinical aspects. In Advances in Cancer Biomarkers (pp. 247-260). Springer, Dordrecht. 
Schiffman JD, PG Fisher, and P Gibbs (2015) Early detection of cancer: past, present, and future. AmSocClinOncolEduc Book 35(1):57-65.

Siama Z, M Zosang-zuali, A Vanlalruati, GC Jagetia, KS Pau, and NS Kumar (2019) Chronic low dose exposure of hospital workers to ionizing radiation leads to increased micronuclei frequency and reduced antioxidants in their peripheral blood lymphocytes. Int $\mathbf{J}$ Radiat Biol 95(6): 697-709.

Taeb S, SM Mortazavi, A Ghaderi, H Mozdarani, CE de Almeida, MR Kardan, SA Mortazavi, A Soleimani, I Nikokar, M Haghani, and A Soofi (2014) Alterations of PSA, CA15. 3, CA125, Cyfra21-1, CEA, CA19. 9, AFP and Tag72 tumor markers in human blood serum due to long term exposure to high levels of natural background radiation in Ramsar, Iran. Int. J. Radiat. Res 12(2): 133-138.
Till JE, and H Grogan (2008) Radiological risk assessment and environmental analysis. Oxford University Press.

Wannhoff A, M Brune, J Knierim, KH Weiss, C Rupp, and DN Gotthardt (2019) Longitudinal analysis of CA19-9 reveals individualized normal range and early changes before development of biliary tract cancer in patients with primary sclerosing cholangitis. Aliment PharmacolTher 49(6): 769-778

World Health Organization (2003) Occupational radiation protection: Protecting workers against exposure to ionizing radiation. Proceedings of an international conference, Geneva, 26-30 August 2002. International Atomic Energy Agency. Accessed at: https://wwwpub.iaea.org/MTCD/publications/PDF/Pub1145_web.pdf. 